\title{
Functional approaches to lifespan development - Editorial
}

\author{
Röcke, Christina ; Jäncke, Lutz ; Martin, Mike
}

\begin{abstract}
Most of lifespan research focuses on age-related changes and plasticity as early markers of decline or performance improvements achieved through training interventions. This special issue on functional approaches to lifespan development focuses on age-related stabilization in complex functions such as cognitive and motor performance and well-being on the levels of brain and behavior. One central question focuses on what elements have to change for individually important outcomes to remain stable in old age, putting the many experimental studies on the plasticity of elementary processes in the context of the recognition that aging individuals most often successfully manage their development and maintain high levels of functioning. Aging research will profit from understanding the processes and potentials of lifespan developmental stabilization.
\end{abstract}

DOI: https://doi.org/10.1024/1662-9647/a000069

Posted at the Zurich Open Repository and Archive, University of Zurich ZORA URL: https://doi.org/10.5167/uzh-68927

Journal Article

Originally published at:

Röcke, Christina; Jäncke, Lutz; Martin, Mike (2012). Functional approaches to lifespan development Editorial. GeroPsych, 25(4):185-188.

DOI: https://doi.org/10.1024/1662-9647/a000069 
Running head: Functional Approaches to Lifespan Development

FUNCTIONAL APPROACHES TO LIFESPAN DEVELOPMENT: TOWARDS AGING RESEARCH AS THE SCIENCE OF STABILIZATION

\author{
Mike Martin ${ }^{1,2}$ \\ Lutz Jäncke ${ }^{1,3}$ \\ Christina Röcke ${ }^{3}$
}

University of Zürich, Switzerland

Psychologisches Institut ${ }^{1}$

Zentrum für Gerontologie ${ }^{2}$

International Normal Aging and Plasticity Imaging Center (INAPIC) $^{3}$

In press, GeroPsych

Address for correspondence:

Mike Martin

University of Zurich

Zentrum für Gerontologie

Sumatrastrasse 30

CH-8006 Zürich

Phone: +41446357421

Fax: +41446357419

E-Mail:m.martin@psychologie.uzh.ch 


\begin{abstract}
Most of lifespan research focuses on age-related changes and plasticity in terms of early markers of decline or performance improvements through training interventions. This special issue on functional approaches to lifespan development focuses on age-related stabilization in complex functions such as cognitive and motor performance and wellbeing on the levels of brain and behavior. One central question focuses on what elements have to change for individually important outcomes to remain stable in old age, putting the many experimental studies on the plasticity of elementary processes in the context of recognizing that aging individuals most often successfully manage their development and maintain high levels of functioning. Aging research will profit from understanding the processes and potentials of lifespan developmental stabilization.
\end{abstract}


By definition, theoretical approaches of lifespan development are trying to predict and explain both, age-related changes and age-related stability. However, most of lifespan research focuses on age-related changes and plasticity, either by searching for early markers and predictors of decline or by examining the performance improvements through training interventions. Thus, current aging research can be mainly characterized as the science of change. This special issue on functional approaches to lifespan development focuses on possibilities of age-related stabilization in complex functions such as cognitive and motor functioning, everyday performance, wellbeing and emotional functioning, identity, quality of life, quality of relationships, health or control beliefs on the levels of the brain and behavior.

\section{The concept of developmental stabilization}

This focus reflects the phenomenon that in many cases in everyday life individuals recruit, sequence and orchestrate simultaneously multiple abilities and resources to stabilize individually prioritized complex outcomes. By stabilization we thus refer to a dynamic process unfolding within individuals over different time periods, rather than the more outcome-focused term ,stability“. The concept of stabilization is related to the notion of dynamic equilibrium and dynamic equilibrium states that represent flexible responses to changes in environmental demands in relation to available resources proposed within Dynamics Systems Modeling and recently integrated into a theoretical model of adult cognitive plasticity (Lövdén, Bäckman, Lindenberger, Schäfer, \& Schmiedek, 2010) rather than inflexible and static phenomena. Stabilization is different from the notion of continuity proposed in the child as well as aging developmental literature because it is not merely the persistence (vs. qualitative change) of certain features and organizations in a given function or domain from one age period to another (e.g., Kagan, 2008) nor limited to strategies used to ensure the maintenance of internal characteristics and social behaviors (Atchley, 1989, but see also Atchley \& Scala, 1998). Both of these alternative constructs are limited in the scope of 
application and do not explicitly focus on the short- and long-term dynamic processes of stability and change between lower- and higher order functions related to environmental demands, available resources and personal goals.

Our central rationale is that orchestration processes inherent in the dynamical stabilization process may differ between individuals but also depend on the functional domain and the individual mean level. Stabilization may or may not include a focus on improvement over the short-term to ensure stabilization of a higher order functioning over the long run. In the case of palliative care it is, for example, the declared aim to stabilize quality of life for as long as possible, and care providers search for the individually optimal stabilization support. In addition, age-related motivational shifts from prioritizing improvement goals to maintenance and prevention of loss goals have been well-documented in the aging and motivation literature (Ebner, Freund, \& Baltes, 2006) and individuals perceive and evince a great deal of self-related stability in such higher order functions as life satisfaction and affect (Röcke \& Lachman, 2008; Röcke, Li, \& Smith, 2009). However, very little is known by exactly which rules this orchestration is governed and very little research aiming for evidence on the stabilizing effects of interventions but also intervention-independent longitudinal stabilization exists. By encouraging authors to face the methodological challenge of proving that specific changes in elementary processes being part of the orchestration lead to stability of a higher order function - possibly via short-term changes at a lower-level - they have come up with a variety of new and testable models of stabilization. We believe that this lays the groundwork for an evidence base of individualized stabilization interventions that will necessarily be different from change-oriented interventions. What is more, asking what elements have to change for individually important outcomes to remain stable in old age puts the many experimental studies on the plasticity of elementary processes in old age in the context of recognizing that aging individuals most often successfully manage their own development, and that this management allows to maintain high levels of functioning. This 
suggests that in addition to understanding what may lead to malfunctioning or illness, and what may help to improve after an illness, aging research will profit from understanding the processes and potentials of developmental stabilization across the lifespan.

\section{Functional approaches to lifespan development in psychology}

Functional approaches to development have a long tradition within psychology (e.g., Hultsch \& Hertzog, 1988). As Boring (1957) defined it, functional psychology is "success in living, with the adaptation of the organism to its environment, and with the organism's adaptation of its environment to itself" (p. 551). Thus, functional approaches aim at explaining how individuals through the variation in the recruitment of multiple elementary subprocesses manage to adapt to varying environmental conditions and contingencies. Hultsch and Hertzog (1988; see also Dixon \& Hertzog, 1988) point out three central implications of this approach, which also represent key elements of contemporary lifespan developmental theory and research: (a) a focus on the active individual orchestrating multiple subprocesses such as memory retrieval and mnemonic strategies to achieve a stable performance in the higher-order process of intentional behavior, (b) a focus on the dynamic, temporal nature of mental and behavioral activity, and (c) the need to examine psychological operations as they occur under actual living conditions (e.g., Verhaeghen, Martin, \& Sedek, 2012).

As a consequence, if success in living is defined as achieving person-environment-fit in varying environmental conditions, the research target for functional approaches is the process of (functional) stabilization itself. In fact, when Piaget (1936) uses the term "equilibration" to describe development across childhood, he is referring to this stabilization process. What is more, assuming that this stabilization results in a stable level of higher-order outcomes such as identity or wellbeing, then developmental changes or plasticity in the stabilization process are required to achieve stability in such outcomes (e.g., Zinke, Zeintl, 
Eschen, Hertzog, \& Kliegel, 2012; Zöllig, \& Eschen, 2009). Successful lifespan development, it follows, would lead to stable outcomes such as in identity, wellbeing or cognitive and physical health. Thus, using correlational research designs that examine risk and protective factors for variance in wellbeing (or any other of these higher-order outcomes) can tell us about risk and protective factors for unsuccessful developmental stabilization. However, these predictors are unlikely to be identical to factors contributing to the stabilization in successful stabilizers. As the complete breakdown of developmental stabilization, illustrated by the number of individuals with clinical symptoms across the lifespan, is more rare than the successful stabilization, we argue that it would be productive for aging research in general to distinguish between lifespan developmental research focusing on predictors of both positive and negative destabilization (i.e., gains and losses) and lifespan developmental research focusing on predictors of stabilization. Whereas the first may identify single or accumulative risk and protective factors and may lay the groundwork for early detection and prevention (e.g., Eschen, Martin, Schreiter Gasser, \& Kliegel, 2009; Schmid, Eschen, Rüegger-Frey, \& Martin, 2012), the second must have a concept of and can empirically test the essential elements and longitudinal short-term within-person dynamics of the longer-term stabilization of a particular goal function, thus laying the groundwork for truly maintaining higher-order functions across the lifespan. Generally speaking, whenever a higher-order function is potentially stabilized through the dynamic engagement of two or more nonlinear dynamic systems and the level of the goal function feeds back into the regulation of the nonlinear dynamic systems, the functional approach can potentially be applied.

\section{An example application of a functional approach: The fQOL model}

To illustrate our understanding of a functional approach to lifespan development, we have recently introduced the concept of functional Quality of Life (fQOL; Martin, Schneider, Eicher, \& Moor, 2012). In this model, key elements required for life quality stabilization 
include a) functionality representations of specific resources, b) goal-related activities, c) goal domains, and d) the interrelations within and between these elements. One central premise of the model is that a greater representation of resources as being highly functional for the performance of different complex activities that are instrumental for the pursuit of individually meaningful goals is related to higher $f \mathrm{QOL}$ The fQOL approach proposes four different ways to improve life quality, (a) adapting resource functionality assessments, (b) adapting activity complexity (requiring more different functional resources) and overlap (reducing activity contradictions and potential overload), (c) adapting the number and heterogeneity of goals, and (d) adapting the dynamics of adaptation (from passive responding to resource changes to proactive processes of activity development and multiple goal management). As a consequence, the complex intraindividual dynamics behind QOLstabilization are not only described but also made accessible to empirical testing and intervention-development.

One key element of the model is its specification of individual fQOL such that, on the one hand, interindividually different resources and strategies can be identified and examined, which can, on the other hand, be explained by the same principles of individual functionality. Second, and in contrast to most approaches in the aging literature which focus on selfregulation as coping or overcoming critical life events or increasing impairments, the functional approach can be applied to self-initiated changes in its constituting elements in the absence of resource impairments and thus generates new hypotheses that are very different from predictions made within the more classical change-focused models.

Generally speaking, the $f$ QOL approach does not predict a strong association between objectively available resources and subjective ratings of one's quality of life, even though these two aspects are undoubtedly related (cf. Boker \& Martin, in press; Martin \& Moor, 2012). Rather, given individuals can be characterized by a diverse set of simultaneously effective characteristics, these multidimensional and goal-oriented individuals can be 
considered as managers of their own life. As a consequence, QOL for a given individual may remain stable even when faced with decline in other domains such as health, because (a) more than one factor such as physical abilities is needed to stabilize QOL, and (b) multiple constellations of resource levels may equally stabilize QOL. In our view it is thus crucial to build theories about the processes underlying the maintenance of subjective quality of life to improve our understanding of individual differences in general, because these processes and rules may be identically relevant to explain individual similarities (Martin \& Moor, 2012).

Comparable to this illustration most authors in this special issue focus on higher-order behavioral functions such as cognition, motor functioning, emotional well-being and quality of life, personality, control beliefs and interactions among these domains. However, the functional approach to development is equally applicable to the dynamic recruitment of neural structures to achieve stability in more complex outcomes (e.g., Imfeld, Oechslin, Meyer, Loenneker, \& Jäncke, 2009; Munte, Altenmüller, \& Jäncke, 2002). In this context, consider that as individuals age, they may have to recruit neural networks differently to achieve the same performance outcome (e.g., Bezzola, Mérillat, \& Jäncke, 2012; Hayes \& Cabeza, 2008; Willis, Schaie, \& Martin, 2009; Zöllig et al., 2012). The approach may also be applied on the levels of genetics, cells, organs, individuals, and societies. On each level, the matching research strategies are identical. That is, as a minimum for each function it needs to be defined (a) which are the essential contributing systems, (b) which are ranges of activity for the contributing systems (i.e., their plasticity), (c) which are the stabilization parameters for each system, and (c) which rules govern the feedback between the function and the contributing systems. It then needs to be (d) demonstrated which contributing systems are required for the stabilization. Finally, it is possible to (e) develop and test stabilizing interventions.

Using this step-wise procedure, the theoretical models for each level and for each function need to be specified. In turn, appropriate research designs and analytical approaches 
need to be implemented to both examine short-term changes as well as long-term stabilization, both with and without a previous intervention. The analytical methodology to examine the dynamics of stabilization has the potential to become the lingua franca of lifespan developmentalists from different fields and disciplines if researchers familiarize themselves with the core methodological concepts and begin to better integrate recent methodological advances from both classical longitudinal analysis approaches as well as the more brainrelated neuroimaging methods literature. In each field, the focus on the dynamics of stabilization opens a range of new research questions and complements the search for the predictors of system breakdowns as well as improvements. The operationalization of stabilization may be an interesting challenge and differ depending on the functional domain under focus, ranging from the short-term dynamics of emotional regulation and coping strategies focused at the long-term stabilization of well-being and satisfaction with life to the dynamics of brain functioning related to the stabilization of a certain cognitive function. In addition, the more researchers from different fields think about how individually central higher-order functions are stabilized across the lifespan, the more they contribute to a differential image of aging individuals in that the research model of age-related decline and plasticity is complemented by a research model of age-related stability in complex goal functions that can and must be studied in real life. 


\section{References}

Atchley, R. C. (1989). A continuity theory of normal aging. Gerontologist, 29, 183-190.

Atchley, R. C., \& Scala, M. A. (1998). Long-range antecedents of functional capability in later life. Journal of Aging Health, 10, 3-19.

Bezzola, L., Mérillat, S., Jäncke, L. (2012). The effect of leisure activity golf practice on motor imagery: an fMRI study in middle adulthood. Frontiers in Human Neuroscience, 6:67. doi: 10.3389/fnhum.2012.00067.

Boker, S. M. \& Martin, M. (in press). On the Equilibrium Dynamics of Meaning. In M. Edwards \& R. MacCallum (Eds), Current Issues in the Theory and Application of Latent Variable Models. New York: Taylor \& Francis.

Boring, E. G. (1957). A History of Experimental Psychology (2nd ed). Englewood Cliffs, NJ: Prentice-Hall.

Dixon, R., \& Hertzog, C. (1988). A functional approach to memory and metamemory development in adulthood. In F. E. Weinert \& M. Perlmutter (Eds.), Memory development: Universal changes and individual differences (pp. 293-339). Hillsdale, NJ: Lawrence Erlbaum.

Ebner, N. C., Freund, A. M., \& Baltes, P. B. (2006). Developmental changes in personal goal orientation from young to late adulthood: From striving for gains to maintenance and prevention of losses. Psychology and Aging, 21, 664-678.

Eschen, A., Martin, M., Schreiter Gasser, U., \& Kliegel, M. (2009). Prospective and retrospective memory complaints in Mild Cognitive Impairment and mild Alzheimer's disease. Brain Impairment, 10, 59-75.

Hayes, S. M. \& Cabeza, R. (2008). Imaging aging: Present and future. In S. M. Hofer \& D. F. Alwin (Eds.), Handbook of cognitive aging: Interdisciplinary perspectives (pp. 308326). Los Angeles, CA: Sage. 
Hultsch, \& Hertzog, C. (1988). Adult memory and metamemory development. In F. E. Weinert \& M. Perlmutter (Eds.), Memory Development (pp. 292-322). Hillsdale, NJ: Erlbaum.

Imfeld, A., Oechslin, M. S., Meyer, M., Loenneker, T., \& Jäncke, L. (2009). White matter plasticity in the corticospinal tract of musicians: A diffusion tensor imaging study. Neuroimage, 46, 600-607.

Kagan, J. (2008). In defense of qualitative changes in development. Child Development, 79, $1606-1624$.

Martin, M., \& Moor, C. (2012). How psychology as a discipline can profit from focusing psychological research on the individual. European Psychologist, 17, 28-33.

Martin, M., Schneider, R., Eicher, S., \& Moor, C. (2012). The functional Quality of Life ( $f \mathrm{QOL})$ model: A new basis for Quality of life-enhancing interventions in old age. Journal of Gerontopsychology and Geriatric Psychiatry, 25(1), 33-40.

Munte, T. F., Altenmüller, E., \& Jäncke, L. (2002). The musician's brain as a model of neuroplasticity. Nature Review Neuroscience, 3, 473-478.

Willis, S. L., Schaie, K. W., \& Martin, M. (2009). Cognitive plasticity. In V. Bengtson, M. Silverstein, N. Putney, \& D. Gans (Eds.), Handbook of Theories of Aging (pp. 295322). New York: Springer.

Piaget, J. (1936). Origins of Intelligence in the Child. London: Routledge \& Kegan Paul.

Röcke, C., \& Lachmann, M. E. (2008). Perceived trajectories of life satisfaction across past, present, and future: Profiles and correlates of subjective change in young, middle-aged, and older adults. Psychology and Aging, 23, 833-847.

Röcke, C., Li, S.-C., \& Smith, J. (2009). Intraindividual variability in positive and negative affect over 45 days: Do older adults fluctuate less than young adults? Psychology and Aging, 24, 863-878. 
Schmid, R., Eschen, A., Rüegger-Frey, B., \& Martin, M. (2012). Instruments for comprehensive needs assessment in individuals with cognitive complaints, mild cognitive impairment or dementia: A systematic review. International Journal of Geriatric Psychiatry, 27, 329-341.

Verhaeghen, P., Martin, M., \& Sedek, G. (2012). Reconnecting cognition in the lab and cognition in real life: The role of compensatory social and motivational factors in explaining how cognition ages in the wild. Aging, Neuropsychology, and Cognition, 19(1-2), 1-12.

Zinke, K., Zeintl, M., Eschen, A., Hertzog, C., \& Kliegel, M. (2012). Potentials and limits of plasticity induced by a working memory training in old-old age. Gerontology, 58, 7987.

Zöllig, J., \& Eschen, A. (2009). Measuring compensation and its plasticity across the lifespan. Restorative Neurology and Neuroscience, 27, 421-433.

Zöllig, J., Mattli, F., Sutter, C., Aurelio, A., \& Martin, M. (2012). Plasticity of prospective memory through a familiarization intervention in old adults. Aging, Neuropsychology and Cognition, 19, 168-194. 